\title{
Zirconium-Catalyzed Enantioselective [3+2] Cycloaddition of Hydrazones to Olefins Leading to Optically Active Pyrazolidine, Pyrazoline, and 1,3-Diamine Derivatives
}

\author{
Yasuhiro Yamashita and Shū Kobayashi* \\ Graduate School of Pharmaceutical Sciences, The University of Tokyo, \\ Hongo, Bunkyo-ku, Tokyo 113-0033 Japan

\section{Supporting Information}

Table of Contents

Pages

General

S-1

Typical experimental procedure

S-2

Transformation of the products

$\mathrm{S}-12$

References

S-18

\section{Experimental Section}

\section{General}

${ }^{1} \mathrm{H}$ and ${ }^{13} \mathrm{C}$ NMR spectra were recorded on a JEOL JNM-LA300, JNM-LA400, or JNM-LA500 spectrometer in $\mathrm{CDCl}_{3}$, unless otherwise noted. Tetramethylsilane (TMS) served as internal standard $(0 \mathrm{ppm})$ for ${ }^{1} \mathrm{H} \mathrm{NMR}$, and $\mathrm{CDCl}_{3}$ was used as internal standard (77.0 ppm) for ${ }^{13} \mathrm{C}$ NMR. IR spectra were measured with a JASCO FT/IR610 spectrometer. Optical rotations were measured with a JASCO P-1010 polarimeter. 
High-performance liquid chromatography was carried out using following apparatuses; SHIMADZU LC-10AT (liquid chrmatograph), SHIMADZU SPD-10A (UV detecter), and SHIMADZU C-R6A Chromatopac. EI high-resolution mass spectra (EI-HRMS) were measured with JEOL JMX-SX-102 Mass Spectrometer. ESI high-resolution mass spectra (ESI-HRMS) were measured with Bruker Daltonics BioTOF II. Column chromatography was conducted on Silica gel 60 (Merk) or Aluminium Oxide (activated, about 300 mesh, Wako) and preparative thin-layer chromatography was carried out using Wakogel B-5F. All solvents were distilled and dried over MS 4A. Zirconium propoxide-propanol complex $\left(\mathrm{Zr}(\mathrm{OPr})_{4}\right)$ was purchased from Fluka Chemie AG. Propanol was distilled in the presence of magnesium propoxide. BINOLs were synthesized according to the literatures. ${ }^{1}$ Ketene dimethyl dithioacetal $2 \mathbf{2 a}$ was synthesized according to the literature's method. ${ }^{2}$ Vinyl ethers were purchased from Sigma-Aldrich Co. and distilled just before use. All hydrazones were prepared by mixing appropriate aldehydes and hydrazines in DMF or THF at room temperature in the presence of a small amount of aqueous hydrochloric acid, and were purified by recrystallization. All reactions were carried out under argon atmosphere in well-dried glassware.

\section{Typical experimental procedures for asymmetric intermolecular [3+2]} cycloaddition of hydrazones with olefins using chiral zirconium catalysts: Typical experimental procedures are described for the reactions of $\mathbf{1 b}$ with $\mathbf{2 a}$ and of $\mathbf{1 a}$ with $\mathbf{2 c}$.

Asymmetric cycloaddition of $1 \mathbf{b}$ with $2 \mathbf{a}$ : To a suspension of $(R)-3,3^{\prime}-\mathrm{I}_{2} \mathrm{BINOL}$ (3b, $0.048 \mathrm{mmol})$ in toluene $(0.3 \mathrm{~mL})$ was added $\mathrm{Zr}(\mathrm{OPr})_{4}(0.040 \mathrm{mmol})$ in toluene $(0.4$ $\mathrm{mL}$ ) at room temperature. The mixture was stirred for $0.5 \mathrm{~h}$ at the same temperature, 
and propanol $(0.2 \mathrm{mmol})$ in toluene $(0.3 \mathrm{~mL})$ was added. The mixture was stirred for additional $0.5 \mathrm{~h}$. The catalyst solution was transferred to another vessel using toluene $(0.5 \mathrm{~mL})$, in which hydrazone $\mathbf{1 b}(0.40 \mathrm{mmol})$ was placed, and the mixture was stirred at $0{ }^{\circ} \mathrm{C}$. Ketene acetal $\mathbf{2 a}$ in toluene $(0.5 \mathrm{~mL})$ was then added to the suspension, and the whole was stirred at the same temperature for $18 \mathrm{~h}$. After water was added to quench the reaction, the mixture was extracted three times with $\mathrm{CH}_{2} \mathrm{Cl}_{2}$. The organic phases were combined and dried over anhydrous $\mathrm{Na}_{2} \mathrm{SO}_{4}$. After filtration and concentration under reduced pressure, the crude product was purified by column chromatography (aluminium oxide) to afford the desired pyrazolidine derivative (4ba). The optical purity of this adduct was determined by HPLC analysis using a chiral column.

Asymmetric cycloaddition of 1a with 2c: To a suspension of $(R)-3,3^{\prime}, 6,6^{\prime}-$ $\mathrm{I}_{4} \mathrm{BINOL}(3 \mathbf{a}, 0.048 \mathrm{mmol})$ in toluene $(0.3 \mathrm{~mL})$ was added $\mathrm{Zr}(\mathrm{OPr})_{4}(0.040 \mathrm{mmol})$ in toluene $(0.4 \mathrm{~mL})$ at room temperature. The mixture was stirred for $3 \mathrm{~h}$ at the same temperature. The catalyst solution was transferred to another vessel using toluene $(0.8$ $\mathrm{mL})$, in which hydrazone $\mathbf{1 a}(0.40 \mathrm{mmol})$ was placed, and the mixture was stirred at 0 ${ }^{\circ} \mathrm{C}$. Vinyl ether $2 \mathrm{c}(4.0 \mathrm{mmol})$ in toluene $(0.5 \mathrm{~mL})$ was then added to the suspension, and the whole was stirred at the same temperature for $18 \mathrm{~h}$. After water was added to quench the reaction, the mixture was extracted three times with $\mathrm{CH}_{2} \mathrm{Cl}_{2}$. The organic phases were combined, and dried over anhydrous $\mathrm{Na}_{2} \mathrm{SO}_{4}$. After filtration and concentration under reduced pressure, the crude product was purified by column chromatography (aluminium oxide) to afford the desired pyrazolidine derivative (5ac). The diastereomer ratio was determined by ${ }^{1} \mathrm{H}$ NMR analysis, and the optical purity of this adduct was determined by HPLC analysis using a chiral column. 
1-(p-Nitrobenzoyl)-5,5-bis(methylthio)-3-(2-phenylethyl)pyrazolidine (4aa): $[\alpha]_{D}{ }^{28}$ -5.05 (c 2.49, benzene, 63\% ee); IR [cm-1] (neat) 3209, 1653, 1602, 1522, 1496, 1388, 1344, 1233; ${ }^{1} \mathrm{H}$ NMR $\left(\mathrm{CDCl}_{3}\right) \delta=1.85(\mathrm{~m}, 2 \mathrm{H}), 2.18(\mathrm{dd}, 1 \mathrm{H}, J=13.7,7.8 \mathrm{~Hz}), 2.32(\mathrm{~s}$, 3H), $2.41(\mathrm{~s}, 3 \mathrm{H}), 2.63(\mathrm{~m}, 2 \mathrm{H}), 2.82(\mathrm{dd}, 1 \mathrm{H}, J=13.6,7.1 \mathrm{~Hz}), 3.30(\mathrm{~m}, 1 \mathrm{H}), 4.58(\mathrm{br}$, 1H), $7.1(\mathrm{~m}, 2 \mathrm{H}), 7.2-7.4(\mathrm{~m}, 3 \mathrm{H}), 7.76(\mathrm{~d}, 2 \mathrm{H}, J=9.0 \mathrm{~Hz}), 8.22(\mathrm{~d}, 2 \mathrm{H}, J=9.0 \mathrm{~Hz})$; ${ }^{13} \mathrm{C} \mathrm{NMR}\left(\mathrm{CDCl}_{3}\right) \delta=15.8,15.9,32.8,34.1,51.5,56.8,82.4,122.7,126.2,128.1$, 128.4, 129.2, 140.5, 142.2, 148.2, 165.8; ESI-HRMS (m/z) calcd. for $\mathrm{C}_{20} \mathrm{H}_{23} \mathrm{~N}_{3} \mathrm{O}_{3} \mathrm{~S}_{2} \mathrm{Na}$ $\left(\mathrm{M}+\mathrm{Na}^{+}\right)$: 440.1079; found: 440.1070; HPLC Daicel Chiralpak AD, hexane/ ${ }^{\mathrm{P} r O H}=$ $9 / 1$, flow rate $=0.5 \mathrm{~mL} / \mathrm{min}: t_{\mathrm{R}}=57 \mathrm{~min}\left(\right.$ minor), $t_{\mathrm{R}}=64 \mathrm{~min}$ (major).

1-Benzoyl-5,5-bis(methylthio)-3-(2-phenylethyl)pyrazolidine (4ba): $[\alpha]_{\mathrm{D}}^{22}+1.89(\mathrm{c}$ 1.03, benzene, 97\% ee); IR [ $\left.\mathrm{cm}^{-1}\right]$ (neat) 3207, 1739, 1637, 1602, 1577, 1496, 1444, 1383, 1244; ${ }^{1} \mathrm{H}$ NMR $\left(\mathrm{CDCl}_{3}\right) \delta=1.81(\mathrm{~m}, 1 \mathrm{H}), 1.90(\mathrm{~m}, 1 \mathrm{H}), 2.18(\mathrm{dd}, 1 \mathrm{H}, J=13.6$, $7.6 \mathrm{~Hz}), 2.31(\mathrm{~s}, 3 \mathrm{H}), 2.40(\mathrm{~s}, 3 \mathrm{H}), 2.6-2.7(\mathrm{~m}, 2 \mathrm{H}), 2.80(\mathrm{dd}, 1 \mathrm{H}, J=13.6,6.8 \mathrm{~Hz}), 3.31$ $(\mathrm{m}, 1 \mathrm{H}), 4.62(\mathrm{dbr}, 1 \mathrm{H}, J=10.5 \mathrm{~Hz}), 7.12(\mathrm{~d}, 2 \mathrm{H}, J=7.1 \mathrm{~Hz}), 7.2(\mathrm{~m}, 1 \mathrm{H}), 7.3(\mathrm{~m}, 2 \mathrm{H})$, $7.4(\mathrm{~m}, 3 \mathrm{H}), 7.67(\mathrm{~d}, 2 \mathrm{H}, J=6.6 \mathrm{~Hz}) ;{ }^{13} \mathrm{C} \mathrm{NMR}\left(\mathrm{CDCl}_{3}\right) \delta=15.8,16.0,32.9,34.7$, $51.8,56.6,82.4,126.1,127.4,128.3,128.5,128.6,130.1,136.1,140.9,168.1$; ESIHRMS (m/z) calcd. for $\mathrm{C}_{20} \mathrm{H}_{25} \mathrm{~N}_{2} \mathrm{OS}_{2}\left(\mathrm{M}+\mathrm{H}^{+}\right)$: 373.1408; found: 373.1398; HPLC Daicel Chiralcel OD, hexane $/ i \mathrm{PrOH}=9 / 1$, flow rate $=0.3 \mathrm{~mL} / \mathrm{min}: t_{\mathrm{R}}=78 \mathrm{~min}$ (major), $t_{\mathrm{R}}=86 \min ($ minor).

1-Benzoyl-5,5-bis(methylthio)-3-(2-methylpropyl)pyrazolidine (4ca): $[\alpha]_{\mathrm{D}}^{30}+7.61$ (c 2.90, benzene, $96 \%$ ee); IR $\left[\mathrm{cm}^{-1}\right]$ (neat) 3205, 1645, 1577, 1468, 1444, 1377, 1317, 1253,$1209 ;{ }^{1} \mathrm{H}$ NMR $\left(\mathrm{CDCl}_{3}\right) \delta=0.89(\mathrm{~d}, 3 \mathrm{H}, J=6.6 \mathrm{~Hz}), 0.91(\mathrm{~d}, 3 \mathrm{H}, J=6.6 \mathrm{~Hz})$, $1.31(\mathrm{ddd}, 1 \mathrm{H}, J=13.6,6.8,6.8 \mathrm{~Hz}), 1.45(\mathrm{ddd}, 1 \mathrm{H}, J=13.6,7.1,7.1 \mathrm{~Hz}), 1.63(\mathrm{~m}, 1 \mathrm{H})$, $2.09(\mathrm{dd}, 1 \mathrm{H}, J=13.4,8.3 \mathrm{~Hz}), 2.32(\mathrm{~s}, 3 \mathrm{H}), 2.40(\mathrm{~s}, 3 \mathrm{H}), 2.85(\mathrm{dd}, 1 \mathrm{H}, J=13.4,6.6$ 
$\mathrm{Hz}), 3.40(\mathrm{~m} .1 \mathrm{H}), 4.46(\mathrm{dbr}, 1 \mathrm{H}, J=11.2 \mathrm{~Hz}), 7.3-7.4(\mathrm{~m}, 3 \mathrm{H}), 7.6-7.7(\mathrm{~m}, 2 \mathrm{H}) ;{ }^{13} \mathrm{C}$ $\operatorname{NMR}\left(\mathrm{CDCl}_{3}\right) \delta=15.8,15.9,22.5,22.6,25.8,41.7,52.2,55.8,82.1,127.3,128.5$, 130.0, 136.1, 167.7; ESI-HRMS (m/z) calcd. for $\mathrm{C}_{16} \mathrm{H}_{24} \mathrm{~N}_{2} \mathrm{OS}_{2} \mathrm{Na}\left(\mathrm{M}+\mathrm{Na}^{+}\right)$: 347.1228; found: 347.1214; HPLC Daicel Chiralcel OD, hexane $/{ }^{i} \mathrm{PrOH}=40 / 1$, flow rate $=1.0$ $\mathrm{mL} / \mathrm{min}: t_{\mathrm{R}}=20 \min \left(\right.$ major), $t_{\mathrm{R}}=24 \min ($ minor).

1-Benzoyl-5,5-bis(methylthio)-3-cyclohexylpyrazolidine (4da): $[\alpha]_{\mathrm{D}}^{30}+26.0$ (c 2.49 , benzene, $95 \%$ ee); IR $\left[\mathrm{cm}^{-1}\right]$ (neat) $3191,1613,1600.1577,1508,1445,1409,1376$, 1290, 1251, 1206; ${ }^{1} \mathrm{H}$ NMR $\left(\mathrm{CDCl}_{3}\right) \delta=0.9-1.6(\mathrm{~m}, 6 \mathrm{H}), 1.6-1.8(\mathrm{~m}, 5 \mathrm{H}), 2.17(\mathrm{dd}, 1 \mathrm{H}$, $J=13.4,9.0 \mathrm{~Hz}), 2.31(\mathrm{~s}, 3 \mathrm{H}), 2.40(\mathrm{~s}, 3 \mathrm{H}), 2.79(\mathrm{dd}, 1 \mathrm{H}, J=13.4,6.8 \mathrm{~Hz}), 3.07(\mathrm{~m}$, 1H), $4.48(\mathrm{~d}, 1 \mathrm{H}, J=11.6 \mathrm{~Hz}), 7.3-7.4(\mathrm{~m}, 3 \mathrm{H}), 7.67(\mathrm{~m}, 2 \mathrm{H}) ;{ }^{13} \mathrm{C} \mathrm{NMR}\left(\mathrm{CDCl}_{3}\right) \delta=$ $15.9,16.0,25.6,25.8,26.2,29.6,30.8,40.8,50.1,62,6,82.3,127.3,128.7,130.1,136.1$, 167.7; ESI-HRMS (m/z) calcd. for $\mathrm{C}_{18} \mathrm{H}_{26} \mathrm{~N}_{2} \mathrm{OS}_{2} \mathrm{Na}\left(\mathrm{M}+\mathrm{Na}^{+}\right)$: 373.1384; found: 373.1394; HPLC Daicel Chiralcel OD, hexane $/ \mathrm{P} \operatorname{PrOH}=40 / 1$, flow rate $=0.5$ $\mathrm{mL} / \mathrm{min}: t_{\mathrm{R}}=42 \min \left(\right.$ major), $t_{\mathrm{R}}=51 \min ($ minor).

1-Benzoyl-5,5-bis(methylthio)-3-pentylpyrazolidine (4ea): $[\alpha]_{\mathrm{D}}^{24}+8.12$ (c 1.47 , benzene, $98 \%$ ee); IR $\left[\mathrm{cm}^{-1}\right]$ (neat) 3206, 1645, 1462, 1377; ${ }^{1} \mathrm{H} \mathrm{NMR}\left(\mathrm{CDCl}_{3}\right) \delta=0.88$ $(\mathrm{t}, 3 \mathrm{H}, J=6.8 \mathrm{H}), 1.3-1.5(\mathrm{~m}, 6 \mathrm{H}), 1.6-1.7(\mathrm{~m}, 2 \mathrm{H}), 2.13(\mathrm{dd}, 1 \mathrm{H}, J=13.4,8.1 \mathrm{~Hz})$, $2.32(\mathrm{~s}, 3 \mathrm{H}), 2.41(\mathrm{~s}, 3 \mathrm{H}), 2.84(\mathrm{dd}, 1 \mathrm{H}, J=13.4,6.8 \mathrm{~Hz}), 3.32(\mathrm{~m}, 1 \mathrm{H}), 4.50(\mathrm{dbr}, 1 \mathrm{H}, J$ $=11.2 \mathrm{~Hz}), 7.3-7.4(\mathrm{~m}, 3 \mathrm{H}), 7.66(\mathrm{~m}, 2 \mathrm{H}) ;{ }^{13} \mathrm{C} \mathrm{NMR}\left(\mathrm{CDCl}_{3}\right) \delta=13.9,15.9,16.0,22.5$, 26.4, 31.6, 32.8, 52.0, 57.6, 82.3, 127.4, 128.6, 130.1, 136.2, 167.9; ESI-HRMS (m/z) calcd. for $\mathrm{C}_{17} \mathrm{H}_{26} \mathrm{~N}_{2} \mathrm{OS}_{2} \mathrm{Na}\left(\mathrm{M}+\mathrm{Na}^{+}\right)$: 361.1384; found: 361.1380; HPLC Daicel Chiralcel OD, hexane $/{ }^{i} \mathrm{PrOH}=40 / 1$, flow rate $=0.5 \mathrm{~mL} / \mathrm{min}: t_{\mathrm{R}}=47$ min $($ major $), t_{\mathrm{R}}=$ $52 \min$ (minor). 
1-Benzoyl-5,5-bis(methylthio)-3-propylpyrazolidine (4fa): $[\alpha]_{\mathrm{D}}{ }^{26}+12.2$ (c 1.77 , benzene, $96 \%$ ee); IR $\left[\mathrm{cm}^{-1}\right]$ (neat) $3205,1645,1577,1441,1376,1251,1211 ;{ }^{1} \mathrm{H}$ $\operatorname{NMR}\left(\mathrm{CDCl}_{3}\right) \delta=0.91(\mathrm{t}, 3 \mathrm{H}, J=7.1 \mathrm{~Hz}), 1.4(\mathrm{~m}, 3 \mathrm{H}), 1.57(\mathrm{~m}, 1 \mathrm{H}), 2.12(\mathrm{dd}, 1 \mathrm{H}, J=$ 13.6, 8.4 Hz), 2.32 (s, 3H), 2.40 (s, 3H), $2.84(\mathrm{dd}, 1 \mathrm{H}, J=13.6,6.8 \mathrm{~Hz}), 3.33(\mathrm{~m}, 1 \mathrm{H})$, $4.51(\mathrm{dbr}, 1 \mathrm{H}, J=11.2 \mathrm{~Hz}), 7.3-7.4(\mathrm{~m}, 3 \mathrm{H}), 7.67(\mathrm{~m}, 2 \mathrm{H}) ;{ }^{13} \mathrm{C} \mathrm{NMR}\left(\mathrm{CDCl}_{3}\right) \delta=13.8$, 15.8, 15.9, 19.9, 34.7, 51.8, 57.2, 82.2, 127.3, 128.5, 130.0, 136.1, 167.8; ESI-HRMS (m/z) calcd. for $\mathrm{C}_{15} \mathrm{H}_{22} \mathrm{~N}_{2} \mathrm{OS}_{2} \mathrm{Na}\left(\mathrm{M}+\mathrm{Na}^{+}\right)$: 333.1071; found: 333.1080; HPLC Daicel Chiralpak AD, hexane $/{ }^{i} \mathrm{PrOH}=40 / 1$, flow rate $=0.5 \mathrm{~mL} / \mathrm{min}: t_{\mathrm{R}}=50 \mathrm{~min}($ minor $), t_{\mathrm{R}}=$ 56 min (major).

1-Benzoyl-5,5-bis(methylthio)-3-benzylpyrazolidine $\quad(4 \mathrm{ga}):[\alpha]_{\mathrm{D}}{ }^{27}+5.46$ (c 2.84 , benzene, 97\% ee); IR [cm-1] (neat) 3205, 1644, 1601, 1577, 1496, 1448, 1377, 1241; ${ }^{1} \mathrm{H} \mathrm{NMR}\left(\mathrm{CDCl}_{3}\right) \delta=2.26(\mathrm{~s}, 3 \mathrm{H}), 2.26(\mathrm{~m}, 1 \mathrm{H}), 2.36(\mathrm{~s}, 3 \mathrm{H}), 2.65(\mathrm{dd}, 1 \mathrm{H}, J=13.7$, $6.8 \mathrm{~Hz}), 2.69(\mathrm{dd}, 1 \mathrm{H}, J=13.9,7.6 \mathrm{~Hz}), 2.94(\mathrm{dd}, 1 \mathrm{H}, J=13.9,6.6 \mathrm{~Hz}), 3.59(\mathrm{~m}, 1 \mathrm{H})$, $4.75(\mathrm{dbr}, 1 \mathrm{H}, J=9.5 \mathrm{~Hz}), 7.10(\mathrm{~d}, 2 \mathrm{H}, J=6.6 \mathrm{~Hz}), 7.2-7.4(\mathrm{~m}, 6 \mathrm{H}), 7.67(\mathrm{~m}, 2 \mathrm{H}) ;{ }^{13} \mathrm{C}$ $\operatorname{NMR}\left(\mathrm{CDCl}_{3}\right) \delta=15.6,15.8,38.2,50.5,58.1,82.2,126.3,127.1,128.3,128.4,128.7$, 129.8, 135.8, 137.3, 167.7; ESI-HRMS (m/z) calcd. for $\mathrm{C}_{19} \mathrm{H}_{22} \mathrm{~N}_{2} \mathrm{OS}_{2} \mathrm{Na}\left(\mathrm{M}+\mathrm{Na}^{+}\right)$: 381.1071; found: 381.1066; HPLC Daicel Chiralpak AD, hexane $/{ }^{i} \mathrm{PrOH}=9 / 1$, flow rate $=1.0 \mathrm{~mL} / \mathrm{min}: t_{\mathrm{R}}=16 \min ($ minor $), t_{\mathrm{R}}=21 \mathrm{~min}($ major $)$.

\section{1-Benzoyl-5,5-bis(methylthio)-3-(2-(tert-butyldimethylsilyloxy)ethyl)pyrazolidine}

(4ha): $[\alpha]_{\mathrm{D}}{ }^{29}-6.71$ (c 3.54, benzene, $97 \%$ ee); IR $\left[\mathrm{cm}^{-1}\right]$ (neat) 3201, 1649, 1577, 1468, 1444, 1423, 1387, 1254, 1200; ${ }^{1} \mathrm{H}$ NMR $\left(\mathrm{CDCl}_{3}\right) \delta=0.00(\mathrm{~s}, 3 \mathrm{H}), 0.02(\mathrm{~s}, 3 \mathrm{H}), 0.87$ (s, 9H), $1.7(\mathrm{~m}, 2 \mathrm{H}), 2.24(\mathrm{dd}, 1 \mathrm{H}, J=13.4,7.8 \mathrm{~Hz}), 2.31(\mathrm{~s}, 3 \mathrm{H}), 2.39$ (s, 3H), 2.83 (dd, $1 \mathrm{H}, J=13.4,6.8 \mathrm{~Hz}), 3.51(\mathrm{~m}, 1 \mathrm{H}), 3.63(\mathrm{tbr}, 2 \mathrm{H}, J=10.5 \mathrm{~Hz}), 4.71(\mathrm{~d}, 1 \mathrm{H}, J=6.6 \mathrm{~Hz})$, 
7.2 - $7.4(\mathrm{~m}, 3 \mathrm{H}), 7.6$ - $7.7(\mathrm{~m}, 2 \mathrm{H}) ;{ }^{13} \mathrm{C} \mathrm{NMR}\left(\mathrm{CDCl}_{3}\right) \delta=-5.6,15.7,15.8,18.0,26.0$, 35.0, 51.3, 54.7, 59.9, 82.1, 127.2, 128.3, 129.8, 136.1, 167.8; ESI-HRMS (m/z) calcd. for $\mathrm{C}_{20} \mathrm{H}_{34} \mathrm{~N}_{2} \mathrm{O}_{2} \mathrm{~S}_{2} \mathrm{SiNa}\left(\mathrm{M}+\mathrm{Na}^{+}\right)$: 449.1729; found: 449.1725; HPLC Daicel Chiralpak $\mathrm{AD}-\mathrm{H}$, hexane $/{ }^{i} \mathrm{PrOH}=100 / 1$, flow rate $=0.5 \mathrm{~mL} / \mathrm{min}: t_{\mathrm{R}}=70 \mathrm{~min}($ minor $), t_{\mathrm{R}}=77$ $\min ($ major).

1-(p-Nitrobenzoyl)-5-ethoxy-3-(2-phenylethyl)pyrazolidine (5ab): IR $\left[\mathrm{cm}^{-1}\right]$ (neat) $3254,1647,1602,1523,1496,1476,1350 ;{ }^{1} \mathrm{H} \mathrm{NMR}\left(\mathrm{CDCl}_{3}\right.$, two diastereomers mixture) $\delta=1.23(\mathrm{t}, 3 \mathrm{H}, J=7.1 \mathrm{~Hz}), 1.3-1.5(\mathrm{~m}, 1 \mathrm{H}), 1.66(\mathrm{ddd}, 0.5 \mathrm{H}, J=13.2,9.5$, $3.4 \mathrm{~Hz}), 1.8-1.9(\mathrm{~m}, 1 \mathrm{H}), 2.0-2.1(\mathrm{~m}, 0.5 \mathrm{H}), 2.21(\mathrm{ddd}, 0.5 \mathrm{H}, J=13.7,8.0,2.7 \mathrm{~Hz}), 2.27$ (br, 1H), 2.5-2.7 (m, 1.5H), $2.89(\mathrm{br}, 0.5 \mathrm{H}), 3.37$ (br, 0.5H), 3.72 (br, 2H), 4.03 (dbr, $0.5 \mathrm{H}, J=12.0 \mathrm{~Hz}), 4.67(\mathrm{dbr}, 0.5 \mathrm{H}, J=3.9 \mathrm{~Hz}), 5.95(\mathrm{br}, 0.5 \mathrm{H}), 6.04(\mathrm{br}, 0.5 \mathrm{H}), 6.86$ (dbr, $1 \mathrm{H}, J=6.8 \mathrm{~Hz}), 7.03(\mathrm{dbr}, 1 \mathrm{H}, J=7.1 \mathrm{~Hz}), 7.1-7.2(\mathrm{~m}, 3 \mathrm{H}), 7.83(\mathrm{~d}, 1 \mathrm{H}, J=8.6$ $\mathrm{Hz}), 7.91(\mathrm{~d}, 1 \mathrm{H}, J=8.8 \mathrm{~Hz}), 8.24(\mathrm{~d}, 1 \mathrm{H}, J=8.5 \mathrm{~Hz}), 8.25(\mathrm{~d}, 1 \mathrm{H}, J=8.5 \mathrm{~Hz}) ;{ }^{13} \mathrm{C}$ $\mathrm{NMR}\left(\mathrm{CDCl}_{3}\right.$, two diastereomers mixture $) \delta=15.1,15.1,32.8,32.9,34.0,35.8,40.2$, $41.2,58.6,60.3,64.5,64.8,86.9,87.7,122.7,125.9,126.1,128.1,128.2,128.3,128.4$, 129.9, 130.1, 140.6, 140.9, 141.0, 148.6, 169.4, 170.2; EI-HRMS (m/z) calcd. for $\mathrm{C}_{20} \mathrm{H}_{23} \mathrm{~N}_{3} \mathrm{O}_{4}\left(\mathrm{M}^{+}\right)$: 369.1689; found: 369.1706; HPLC Daicel Chiralpak AD-H, hexane $/ i \mathrm{PrOH}=9 / 1$, flow rate $=0.3 \mathrm{~mL} / \mathrm{min}$ : major diastereomer $t_{\mathrm{R}}=61 \mathrm{~min}$ (major), $t_{\mathrm{R}}=66 \min \left(\right.$ minor); minor diastereomer $t_{\mathrm{R}}=46 \min$ (major), $t_{\mathrm{R}}=50 \min ($ minor).

1-(p-Nitrobenzoyl)-5-(1-propoxy)-3-(2-phenylethyl)pyrazolidine (5ac): IR $\left[\mathrm{cm}^{-1}\right]$ (neat) $3255,1641,1600,1523,1344 ;{ }^{1} \mathrm{H} \mathrm{NMR}\left(\mathrm{CDCl}_{3}\right.$, two diastereomers mixture $) \delta=$ $0.94(\mathrm{t}, 3 \mathrm{H}, J=7.3 \mathrm{~Hz}), 1.3-1.5(\mathrm{~m}, 1 \mathrm{H}), 1.61(\mathrm{q}, 2 \mathrm{H}, J=7.1 \mathrm{~Hz}), 1.66(\mathrm{~m}, 0.5 \mathrm{H}), 1.8-$ $2.0(\mathrm{~m}, 1 \mathrm{H}), 2.0-2.1(\mathrm{~m}, 0.5 \mathrm{H}), 2.22(\mathrm{ddd}, 0.5 \mathrm{H}, J=13.7,8.1,2.4 \mathrm{~Hz}), 2.28(\mathrm{br}, 1 \mathrm{H})$, 
$2.59(\mathrm{~m}, 0.5 \mathrm{H}), 2.63(\mathrm{~m}, 1 \mathrm{H}), 2.91(\mathrm{br}, 0.5 \mathrm{H}), 3.62(\mathrm{br}, 2 \mathrm{H}), 4.01(\mathrm{dbr}, 0.5 \mathrm{H}, J=11.4$ $\mathrm{Hz}), 4.65(\mathrm{br}, 0.5 \mathrm{H}), 5.95(\mathrm{br}, 0.5 \mathrm{H}), 6.03(\mathrm{br}, 0.5 \mathrm{H}), 6.86(\mathrm{dbr}, 0.5 \mathrm{H}, J=6.8 \mathrm{~Hz}), 7.04$ $(\mathrm{dbr}, 0.5 \mathrm{H}, J=6.8 \mathrm{~Hz}), 7.1-7.3(\mathrm{~m}, 3 \mathrm{H}), 7.83(\mathrm{~d}, 1 \mathrm{H}, J=8.8 \mathrm{~Hz}), 7.91(\mathrm{~d}, 1 \mathrm{H}, J=8.8$ $\mathrm{Hz}), 8.25(\mathrm{~d}, 1 \mathrm{H}, J=8.8 \mathrm{~Hz}), 8.25(\mathrm{~d}, 1 \mathrm{H}, J=9.0 \mathrm{~Hz}) ;{ }^{13} \mathrm{C} \mathrm{NMR}\left(\mathrm{CDCl}_{3}\right.$, two diastereomers mixture) $\delta=10.6,22.8,22.9,32.8,32.9,34.1,35.8,40.2,41.2,58.6,60.3$, $70.8,71.0,87.0,87.9,122.7,126.0,126.2,128.2,128.3,128.4,128.4,129.9,130.2$, 140.6, 141.0, 141.0, 148.7, 169.4, 170.2; EI-HRMS (m/z) calcd. for $\mathrm{C}_{21} \mathrm{H}_{25} \mathrm{~N}_{3} \mathrm{O}_{4}\left(\mathrm{M}^{+}\right)$: 383.1845; found: 383.1838; HPLC Daicel Chiralpak AD, hexane $/{ }^{i} \mathrm{PrOH}=40 / 1$, flow rate $=0.8 \mathrm{~mL} / \mathrm{min}$ : major diastereomer $t_{\mathrm{R}}=69 \min ($ major $), t_{\mathrm{R}}=88 \min ($ minor $)$; minor diastereomer $t_{\mathrm{R}}=50 \mathrm{~min}$ (minor), $t_{\mathrm{R}}=54 \mathrm{~min}$ (major).

1-(p-Nitrobenzoyl)-5-(tert-butoxy)-3-(2-phenylethyl)pyrazolidine (5ad): IR [ $\left.\mathrm{cm}^{-1}\right]$ (neat) $3250,1636,1601,1521,1391,1353,1235 ;{ }^{1} \mathrm{H} \mathrm{NMR}\left(\mathrm{CDCl}_{3}\right.$, two diastereomers mixture $) \delta=1.31(\mathrm{~s}, 9 \mathrm{H}), 1.2-1.5(\mathrm{~m}, 1.8 \mathrm{H}), 1.64(\mathrm{ddd}, 0.2 \mathrm{H}, J=13.4,8.6,3.0 \mathrm{~Hz})$, $1.80(\mathrm{~m}, 0.2 \mathrm{H}), 1.91(\mathrm{ddd}, 0.8 \mathrm{H}, J=13.4,6.1,3.7 \mathrm{~Hz}), 2.16(\mathrm{ddd}, 0.8 \mathrm{H}, J=13.4,8.1$, $1.2 \mathrm{~Hz}), 2.26(\mathrm{t}, 1.6 \mathrm{H}, J=7.6 \mathrm{~Hz}), 2.52(\mathrm{ddd}, 0.2 \mathrm{H}, J=13.4,7.3,7.3 \mathrm{~Hz}), 2.60(\mathrm{t}, 0.4 \mathrm{H}$, $J=7.8 \mathrm{~Hz}), 2.83(\mathrm{~m}, 0.2 \mathrm{H}), 3.36(\mathrm{~m}, 0.8 \mathrm{H}), 4.07(\mathrm{dbr}, 0.2 \mathrm{H}, J=12.0 \mathrm{~Hz}), 4.74(\mathrm{dbr}$, $0.8 \mathrm{H}, J=5.4 \mathrm{~Hz}), 6.15(\mathrm{dbr}, 0.2 \mathrm{H}, J=4.2 \mathrm{~Hz}), 6.25(\mathrm{dbr}, 0.8 \mathrm{H}, J=5.9 \mathrm{~Hz}), 6.84(\mathrm{dbr}$, 1.6H, $J=7.3 \mathrm{~Hz}), 7.01(\mathrm{dbr}, 0.4 \mathrm{H}, J=6.8 \mathrm{~Hz}), 7.1-7.2(\mathrm{~m}, 3 \mathrm{H}), 7.80(\mathrm{~d}, 0.4 \mathrm{H}, J=8.6$ $\mathrm{Hz}), 7.87(\mathrm{~d}, 1.6 \mathrm{H}, J=8.6 \mathrm{~Hz}), 8.22(\mathrm{~d}, 2 \mathrm{H}, J=8.8 \mathrm{~Hz}) ;{ }^{13} \mathrm{C} \mathrm{NMR}\left(\mathrm{CDCl}_{3}\right.$, two diastereomers mixture) $\delta=28.3,28.4,32.8,32.8,34.2,36.4,41.5,42.7,58.4,60.1,75.0$, $75.1,81.3,81.9,122.6,125.8,126.0,128.1,128.2,128.2,128.3,129.8,130.1,140.7$, 141.0, 141.2, 141.3, 148.5, 168.5, 169.1; EI-HRMS (m/z) calcd. for $\mathrm{C}_{22} \mathrm{H}_{27} \mathrm{~N}_{3} \mathrm{O}_{4}\left(\mathrm{M}^{+}\right)$: 397.2002; found: 397.2006; HPLC Daicel Chiralpak AD, hexane $/{ }^{i} \mathrm{PrOH}=40 / 1$, flow 
rate $=0.8 \mathrm{~mL} / \mathrm{min}$ : major diastereomer $t_{\mathrm{R}}=41 \mathrm{~min}$ (major), $t_{\mathrm{R}}=47 \mathrm{~min}($ minor); minor diastereomer $t_{\mathrm{R}}=26 \mathrm{~min}$ (major), $t_{\mathrm{R}}=32 \mathrm{~min}$ (minor).

1-(p-Nitrobenzoyl)-5-ethylthio-3-(2-phenylethyl)pyrazolidine (5ae): IR [ $\left.\mathrm{cm}^{-1}\right]$ (neat) $3251,1643,1601,1521,1496,1453,1349,1219 ; ;{ }^{1} \mathrm{H} \mathrm{NMR}\left(\mathrm{CDCl}_{3}\right.$, two diastereomers mixture) $\delta=1.33(\mathrm{t}, 0.2 \mathrm{H}, J=7.3 \mathrm{~Hz}), 1.35(\mathrm{t}, 0.8 \mathrm{~Hz}, J=7.6 \mathrm{~Hz}), 1.3-1.5(\mathrm{~m}, 2 \mathrm{H})$, $1.84(\mathrm{~m}, 0.2 \mathrm{H}), 1.98(\mathrm{~m}, 0.8 \mathrm{H}), 2.23(\mathrm{~m}, 0.8 \mathrm{H}), 2.27(\mathrm{tbr}, 1.6 \mathrm{H}, J=7.8 \mathrm{~Hz}), 2.6-2.8(\mathrm{~m}$, 1.8H), 2.8-3.0 (m, 1.2H), $3.29(\mathrm{~m}, 0.8 \mathrm{~Hz}), 4.24(\mathrm{dbr}, 0.2 \mathrm{H}, J=12.4 \mathrm{~Hz}), 4.91(\mathrm{dbr}$, $0.8 \mathrm{H}, J=4.9 \mathrm{~Hz}), 5.93(\mathrm{dd}, 0.2 \mathrm{H}, J=8.3,5.6 \mathrm{~Hz}), 6.04(\mathrm{dd}, 0.8 \mathrm{H}, J=8.0,4.4 \mathrm{~Hz}), 6.86$ $(\mathrm{d}, 1.6 \mathrm{H}, J=7.1 \mathrm{~Hz}), 7.04(\mathrm{~m}, 0.4 \mathrm{~Hz}), 7.1-7.3(\mathrm{~m}, 3 \mathrm{H}), 7.83(\mathrm{~d}, 0.4 \mathrm{H}, J=8.8 \mathrm{~Hz}), 7.91$ $(\mathrm{d}, 1.6 \mathrm{H}, J=8.8 \mathrm{~Hz}), 8.2-8.3(\mathrm{~m}, 2 \mathrm{H}) ;{ }^{13} \mathrm{C} \mathrm{NMR}\left(\mathrm{CDCl}_{3}\right.$, two diastereomers mixture $) \delta$ $=14.9,26.1,26.3,32.7,32.9,33.9,35.4,39.8,40.9,58.5,60.1,60.3,61.0,122.7,122.7$, $125.9,126.2,128.1,128.2,128.3,128.4,129.9,130.1,140.4,140.8,140.8,148.7,168.5$, 169.6; EI-HRMS (m/z) calcd. for $\mathrm{C}_{20} \mathrm{H}_{23} \mathrm{~N}_{3} \mathrm{O}_{3} \mathrm{~S}\left(\mathrm{M}^{+}\right)$: 385.1460; found: 385.1465; HPLC Daicel Chiralpak AD-H, hexane $/ \mathrm{PrOH}=19 / 1$, flow rate $=0.8 \mathrm{~mL} / \mathrm{min}$ : major diastereomer $t_{\mathrm{R}}=48 \mathrm{~min}$ (major), $t_{\mathrm{R}}=55 \mathrm{~min}$ (minor); minor diastereomer $t_{\mathrm{R}}=34 \mathrm{~min}$ (minor), $t_{\mathrm{R}}=41 \min$ (major).

1-(p-Nitrobenzoyl)-5-(1-propoxy)-3-(2-methylpropyl)pyrazolidine (5ic): IR [ $\left.\mathrm{cm}^{-1}\right]$ (neat) $3252,1645,1602,1523,1471,1345,1314 ;{ }^{1} \mathrm{H} \mathrm{NMR}\left(\mathrm{CDCl}_{3}\right.$, two diastereomers mixture $) \delta=0.59(\mathrm{~d}, 1.6 \mathrm{H}, J=6.4 \mathrm{~Hz}), 0.70(\mathrm{~d}, 1.6 \mathrm{H}, J=6.3 \mathrm{~Hz}), 0.81(\mathrm{~d}, 1.4 \mathrm{H}, J=$ $6.6 \mathrm{~Hz}), 0.82(\mathrm{~d}, 1.4 \mathrm{H}, J=6.6 \mathrm{~Hz}), 0.89(\mathrm{t}, 3 \mathrm{H}, J=7.6 \mathrm{~Hz}), 0.98(\mathrm{~m}, 0.4 \mathrm{H}), 1.16(\mathrm{~m}$, 0.6 H), 1.3-1.5 (m, 1H), 1.5-1.7 (m, 2.4H), $1.95(\mathrm{~m}, 0.6 \mathrm{H}), 2.16(\mathrm{~m}, 0.6 \mathrm{H}), 2.57$ (ddd, 0.4H, $J=13.4,7.0,7.0 \mathrm{~Hz}), 2.96(\mathrm{~m}, 0.4 \mathrm{H}), 3.43(\mathrm{br}, 0.6 \mathrm{H}), 3.59(\mathrm{br}, 2 \mathrm{H}), 3.95(\mathrm{dbr}$, $0.4 \mathrm{H}, J=12.0 \mathrm{~Hz}), 4.59(\mathrm{dbr}, 0.6 \mathrm{H}, J=4.4 \mathrm{~Hz}), 5.92(\mathrm{br}, 0.4 \mathrm{H}), 5.95$ (br, 0.6H), 7.83 $(\mathrm{d}, 1 \mathrm{H}, J=8.8 \mathrm{~Hz}), 7.84(\mathrm{~d}, 1 \mathrm{H}, J=8.8 \mathrm{~Hz}), 8.19(\mathrm{dbr}, 2 \mathrm{H}, J=8.0 \mathrm{~Hz}) ;{ }^{13} \mathrm{C} \mathrm{NMR}$ 
$\left(\mathrm{CDCl}_{3}\right.$, two diastereomers mixture $) \delta=10.5,21.6,22.4,22.7,22.8,22.8,25.0,26.1$, $40.3,41.5,41.6,43.1,70.7,70.9,86.9,87.8,122.5,122.6,129.8,129.9,141.0,141.2$, 148.5, 148.5, 169.1, 170.2; EI-HRMS (m/z) calcd. for $\mathrm{C}_{17} \mathrm{H}_{25} \mathrm{~N}_{3} \mathrm{O}_{4}\left(\mathrm{M}^{+}\right)$: 335.1845; found: 335.1851; HPLC Daicel Chiralpak AS, hexane $/{ }^{i} \mathrm{PrOH}=19 / 1$, flow rate $=0.5$ $\mathrm{mL} / \mathrm{min}$ : major diastereomer $t_{\mathrm{R}}=57$ min (minor), $t_{\mathrm{R}}=68$ min (major); minor diastereomer $t_{\mathrm{R}}=26 \min$ (major), $t_{\mathrm{R}}=34 \mathrm{~min}$ (minor).

1-(p-Nitrobenzoyl)-5-(1-propoxy)-3-cyclohexylpyrazolidine $(5 \mathrm{jc}):$ IR $\left[\mathrm{cm}^{-1}\right]$ (neat) 3259, 1645, 1601, 1523, 1473, 1344, 1315; ${ }^{1} \mathrm{H}$ NMR $\left(\mathrm{CDCl}_{3}\right.$, two diastereomers mixture) $\delta=0.5-1.4(\mathrm{~m}, 10 \mathrm{H}), 1.4-1.8(\mathrm{~m}, 6.4 \mathrm{H}), 2.08(\mathrm{br}, 1 \mathrm{H}) .2 .49$ (ddd, $0.4 \mathrm{H}, J=$ 13.4, 7.1, 7.1 Hz), $2.66(\mathrm{ddd}, 0.4 \mathrm{H}, J=18.1,8.5,8.5 \mathrm{~Hz}), 3.01(\mathrm{br}, 0.6 \mathrm{H}), 3.56(\mathrm{br}, 2 \mathrm{H})$, $4.01(\mathrm{dbr}, 0.4 \mathrm{H}, J=12.5 \mathrm{~Hz}), 4.57(\mathrm{br}, 0.6 \mathrm{H}), 5.91(\mathrm{br}, 1 \mathrm{H}), 7.8-7.9(\mathrm{~m}, 2 \mathrm{H}), 8.1-8.2(\mathrm{~m}$, $2 \mathrm{H}) ;{ }^{13} \mathrm{C} \mathrm{NMR}\left(\mathrm{CDCl}_{3}\right.$, two diastereomers mixture $) \delta=10.5,22.7,22.8,25.4,25.5$, $25.6,25.8,26.0,26.1,29.3,29.5,30.4,30.8,37.0,39.3,40.8,41.0,64.2,66.1,70.5$, $70.8,86.9,87.7,122.4,129.8,129.9,140.9,141.3,148.4,148.4,168.9,169.7$; EIHRMS (m/z) calcd. for $\mathrm{C}_{19} \mathrm{H}_{27} \mathrm{~N}_{3} \mathrm{O}_{4}\left(\mathrm{M}^{+}\right)$: 361.2002; found: 361.2002; HPLC Daicel Chiralpak $\mathrm{AD}$ with guard column, hexane $/ \mathrm{PrOH}=30 / 1$, flow rate $=0.5 \mathrm{~mL} / \mathrm{min}$ : major diastereomer $t_{\mathrm{R}}=79 \min \left(\right.$ minor), $t_{\mathrm{R}}=91 \mathrm{~min}$ (major); minor diastereomer $t_{\mathrm{R}}=43 \mathrm{~min}$ (major), $t_{\mathrm{R}}=57 \min$ (minor).

1-(p-Nitrobenzoyl)-5-(1-propoxy)-3-pentylpyrazolidine (5kc): IR [ $\left.\mathrm{cm}^{-1}\right]$ (neat) 3258 , $1647,1602,1523,1470,1344 ;{ }^{1} \mathrm{H}$ NMR $\left(\mathrm{CDCl}_{3}\right.$, two diastereomers mixture $) \delta=0.72$ (br, 1.7H), $0.81(\mathrm{br}, 1.3 \mathrm{H}), 0.89(\mathrm{t}, 3 \mathrm{H}, J=7.3 \mathrm{~Hz}), 0.8-1.3(\mathrm{~m}, 6.4 \mathrm{H}), 1.41(\mathrm{~m}, 0.3 \mathrm{H})$, $1.57(\mathrm{~m}, 2.7 \mathrm{H}), 1.98(\mathrm{~m}, 0.6 \mathrm{H}), 2.14(\mathrm{~m}, 0.6 \mathrm{H}), 2.56(\mathrm{ddd}, 0.4 \mathrm{H}, J=13.9,7.3,7.3 \mathrm{~Hz})$, $2.92(\mathrm{br}, 0.4 \mathrm{H}), 3.31(\mathrm{br}, 0.6 \mathrm{H}), 3.59(\mathrm{br}, 2 \mathrm{H}), 3.98(\mathrm{dbr}, 0.4 \mathrm{H}, J=11.7 \mathrm{~Hz}), 4.60$ (br, 
0.6H), $5.95(\mathrm{br}, 1 \mathrm{H}), 7.84(\mathrm{~m}, 2 \mathrm{H}), 8.19(\mathrm{~d}, 2 \mathrm{H}, J=8.8 \mathrm{~Hz}) ;{ }^{13} \mathrm{C} \mathrm{NMR}\left(\mathrm{CDCl}_{3}\right.$, two diastereomers mixture) $\delta=10.5,13.7,13.8,22.3,22.7,22.8,26.1,26.2,31.3,31.5,32.4$, $34.1,40.1,41.1,59.3,61.1,70.7,70.9,87.0,87.8,122.5,122.6,129.8,129.9,141.0$, 141.2, 148.4, 148.5, 169.1, 170.1; EI-HRMS (m/z) calcd. for $\mathrm{C}_{18} \mathrm{H}_{27} \mathrm{~N}_{3} \mathrm{O}_{4}\left(\mathrm{M}^{+}\right)$: 349.2002; found: 349.2008; HPLC Daicel Chiralpak AD-H, hexane/ ${ }^{i} \operatorname{PrOH}=19 / 1$, flow rate $=0.3 \mathrm{~mL} / \mathrm{min}$ : major diastereomer $t_{\mathrm{R}}=53 \min ($ minor $), t_{\mathrm{R}}=56 \min ($ major $) ;$ minor diastereomer $t_{\mathrm{R}}=39 \min$ (major), $t_{\mathrm{R}}=46 \mathrm{~min}$ (minor).

1-(p-Nitrobenzoyl)-5-(1-propoxy)-3-benzylpyrazolidine (5lc): IR [ $\left.\mathrm{cm}^{-1}\right](\mathrm{KBr}) 3257$, 1631, 1601. 1520, 1415, 1387, 1350, 1317, 1296, 1221; ${ }^{1} \mathrm{H}$ NMR $\left(\mathrm{CDCl}_{3}\right.$, two diastereomers mixture) $\delta=0.93(\mathrm{tbr}, 3 \mathrm{H}, J=7.3 \mathrm{~Hz}), 1.60(\mathrm{~m}, 2 \mathrm{H}), 1.78(\mathrm{~m}, 0.3 \mathrm{H})$, 2.1-2.3 (m, 1.4H), 2.3-2.5 (m, 1.7H), $2.71(\mathrm{dd}, 0.3 \mathrm{H}, J=13.4,7.8 \mathrm{~Hz}), 2.98(\mathrm{dd}, 0.3 \mathrm{H}, J$ $=13.7,5.6 \mathrm{~Hz}), 3.24(\mathrm{br}, 0.3 \mathrm{H}), 3.5-3.8(\mathrm{br}, 2.7 \mathrm{H}), 4.21(\mathrm{dbr}, 0.3 \mathrm{H}, J=11.5 \mathrm{~Hz}), 4.75$ (sbr, 0.7 H), 5.93 (sbr, 0.3H), 6.08 (sbr, 0.7H), 6.77 (sbr, 1.4H), 7.1-7.2 (m, 2.7H), 7.2$7.3(\mathrm{~m}, 0.9 \mathrm{H}), 7.86(\mathrm{~d}, 0.6 \mathrm{H}, J=8.8 \mathrm{~Hz}), 7.92(\mathrm{dbr}, 1.4 \mathrm{H}, J=8.1 \mathrm{~Hz}), 8.2-8.3(\mathrm{~m}, 2 \mathrm{H})$; ${ }^{13} \mathrm{C}$ NMR $\left(\mathrm{CDCl}_{3}\right.$, two diastereomers mixture $) \delta=10.5,22.7,38.1,39.4,40.2,40.5$, $60.7,61.6,70.7,70.9,86.9,87.8,122.6,126.2,126.6,128.1,128.5,128.6,128.7,129.9$, 130.3, 137.1, 138.1, 140.7, 140.8, 148.5, 148.6, 169.1, 169.6; EI-HRMS (m/z) calcd. for $\mathrm{C}_{20} \mathrm{H}_{23} \mathrm{~N}_{3} \mathrm{O}_{4}\left(\mathrm{M}^{+}\right)$: 369.1689; found: 369.1697; HPLC Daicel Chiralpak AS-H, hexane $/ \mathrm{PrOH}=9 / 1$, flow rate $=0.3 \mathrm{~mL} / \mathrm{min}$ : minor diastereomer $t_{\mathrm{R}}=72 \mathrm{~min}$ (major), $t_{\mathrm{R}}=80 \min \left(\right.$ minor); minor diastereomer $t_{\mathrm{R}}=113 \min$ (major), $t_{\mathrm{R}}=139 \min$ (minor).

1-(o, p-Dinitrobenzoyl)-5-(1-propoxy)-3-phenylpyrazolidine (5mc): IR [ $\left.\mathrm{cm}^{-1}\right]$ (neat) 3253, 1666, 1603, 1537, 1489, 1456, 1349, 1249; ${ }^{1} \mathrm{H} \mathrm{NMR}\left(\mathrm{CDCl}_{3}\right.$, two diastereomers mixture) $\delta=1.00(\mathrm{t}, 3 \mathrm{H}, J=7.3 \mathrm{~Hz}), 1.70(\mathrm{tq}, 2 \mathrm{H}, J=7.3,7.3 \mathrm{~Hz}), 2.28(\mathrm{ddd}, 0.5 \mathrm{H}, J=$ 
13.6, 7.8, 2.4 Hz), $2.51(\mathrm{ddd}, 0.5 \mathrm{H}, J=13.9,8.1,2.0 \mathrm{~Hz}), 2.70(\mathrm{ddd}, 0.5 \mathrm{H}, J=13.9,5.6$, 5.6 Hz), 2,93 (ddd, 0.5H, $J=13.4,7.3,7.3 \mathrm{~Hz}), 3.7-3.9(\mathrm{~m}, 2 \mathrm{H}), 4.0-4.2(\mathrm{~m}, 1 \mathrm{H}), 4.58$ (br, 0.5H), $4.90(\mathrm{br}, 0.5 \mathrm{H}), 6.03(\mathrm{~m}, 1 \mathrm{H}), 6.83(\mathrm{~d}, 0.5 \mathrm{H}, J=8.6 \mathrm{~Hz}), 6.98(\mathrm{~d}, 1 \mathrm{H}, J=7.1$ Hz), 7.2-7.4 (m, 4H), $7.70(\mathrm{~d}, 1 \mathrm{H}, J=8.5 \mathrm{~Hz}), 8.20(\mathrm{dd}, 0.5 \mathrm{H}, J=8.3,1.7 \mathrm{~Hz}), 8.50(\mathrm{dd}$, $0.5 \mathrm{H}, J=8.6,1.5 \mathrm{~Hz}), 8.86(\mathrm{sbr}, 0.5 \mathrm{H}), 8.91(\mathrm{sbr}, 0.5 \mathrm{H}) ;{ }^{13} \mathrm{C} \mathrm{NMR}\left(\mathrm{CDCl}_{3}\right.$, two diastereomers mixture) $\delta=10.6,10.6,22.8,22.9,39.8,41.7,61.1,63.6,71.2,71.2,86.9$, 87.6, 119.1, 119.3, 127.0, 127.4, 127.5, 127.7, 128.1, 128.4, 128.5, 128.8, 130.0, 130.1, 137.4, 138.2, 138.7, 139.7, 145.7, 145.9, 147.4, 147.7, 166.3, 166.7; EI-HRMS (m/z) calcd. for $\mathrm{C}_{19} \mathrm{H}_{20} \mathrm{~N}_{4} \mathrm{O}_{6}\left(\mathrm{M}^{+}\right)$: 400.1383; found: 400.1392; HPLC Daicel Chiralcel OD, hexane $/ \mathrm{PrOH}=19 / 1$, flow rate $=1.0 \mathrm{~mL} / \mathrm{min}$ : diastereomer $\mathrm{A} t_{\mathrm{R}}=30 \mathrm{~min}($ major $), t_{\mathrm{R}}=$ $40 \min$ (minor); diastereomer $\mathrm{B} t_{\mathrm{R}}=48 \min$ (minor), $t_{\mathrm{R}}=74 \min$ (major).

1-(p-Nitrobenzoyl)-5-(1-propoxy)-3-(phenylthiomethyl)pyrazolidine (5nc): IR [ $\mathrm{cm}^{-}$ 1] (neat) $3252,1651,1601,1523,1494,1479,1439,1345,1315 ;{ }^{1} \mathrm{H} \mathrm{NMR}\left(\mathrm{CDCl}_{3}\right.$, two diastereomers mixture) $\delta=0.93(\mathrm{t}, 1.8 \mathrm{H}, J=7.6 \mathrm{~Hz}), 0.94(\mathrm{t}, 1.2 \mathrm{H}, J=7.3 \mathrm{~Hz}), 1.61(\mathrm{~m}$, 2H), $1.89(\mathrm{~m}, 0.4 \mathrm{H}), 2.27$ (tbr, 1.2H, $J=4.4 \mathrm{~Hz}), 2.5-2.7(\mathrm{~m}, 1 \mathrm{H}), 2.78(\mathrm{~m}, 0.6 \mathrm{H}), 3.11$ (m, 0.6H), $3.24(\mathrm{br}, 0.4 \mathrm{H}), 3.5-3.7(\mathrm{~m}, 2.6 \mathrm{H}), 4.30(\mathrm{br}, 0.4 \mathrm{H}), 4.83$ (br, 0.6H), 5.95 (br, 0.4H), $6.04(\mathrm{br}, 0.6 \mathrm{H}), 7.1-7.4(\mathrm{~m}, 5 \mathrm{H}), 7.82(\mathrm{~d}, 0.8 \mathrm{H}, J=8.5 \mathrm{~Hz}), 7.89(\mathrm{~d}, 1.2 \mathrm{H}, J=8.6$ $\mathrm{Hz})$, 8.2-8.3 (m, $2 \mathrm{H}) ;{ }^{13} \mathrm{C} \mathrm{NMR}\left(\mathrm{CDCl}_{3}\right.$, two diastereomers mixture $) \delta=10.5,10.6$, $22.8,22.8,35.9,38.4,39.0,39.9,58.4,59.8,70.7,70.9,87.0,87.6,122.7,122.8,126.6$, $127.0,129.0,129.0,129.8,130.0,130.0,130.3,134.9,135.2,140.6,148.7,148.7,169.0$, 169.6; EI-HRMS (m/z) calcd. for $\mathrm{C}_{20} \mathrm{H}_{23} \mathrm{~N}_{3} \mathrm{O}_{4} \mathrm{~S}\left(\mathrm{M}^{+}\right)$: 401.1409; found: 401.1408; HPLC Daicel Chiralpak AD-H, hexane $/ \mathrm{PrOH}=30 / 1$, flow rate $=1.0 \mathrm{~mL} / \mathrm{min}$ : major 
diastereomer $t_{\mathrm{R}}=94 \min \left(\right.$ minor), $t_{\mathrm{R}}=108 \mathrm{~min}$ (major); minor diastereomer $t_{\mathrm{R}}=63 \mathrm{~min}$ (minor), $t_{\mathrm{R}}=67$ min (major).

\section{Transformation of the products}

$N$-(3-Amino-1-(methylthio)-5-phenylpentyl)benzamide (6): To a solution of $4 \mathbf{b a}$ (464 mg, 1.,25 mmol) in well-degassed methanol $(\mathrm{MeOH})(35 \mathrm{~mL})$ was added $\mathrm{SmI}_{2}$ in THF (0.15 M, $50 \mathrm{~mL}, 7.5 \mathrm{mmol})$ at $-78^{\circ} \mathrm{C}$, and the mixture was stirred for $14.5 \mathrm{~h}$ at the same temperature. The reaction solution was then exposed to the air at $-78{ }^{\circ} \mathrm{C}$, and the color of the solution turned yellow from dark green. The solvent was evaporated, and the residue was dissolved in a mixture of $\mathrm{CH}_{2} \mathrm{Cl}_{2}$ and a saturated $\mathrm{NaHCO}_{3}$ solution. The mixture was filtrated with Celite ${ }^{\circledR}$, and the organic phase was separated. The aqueous phase was extracted with $\mathrm{CH}_{2} \mathrm{Cl}_{2}$, and the organic phases were combined and washed with water and brine, and dried over anhydrous $\mathrm{Na}_{2} \mathrm{SO}_{4}$. After filtration and concentration under reduced pressure, the crude product was purified by column chromatography ( silica gel, $\mathrm{CHCl}_{3}: \mathrm{MeOH}=20 ; 1$ ) to afford the desired product as a mixture of diastereomers $(320 \mathrm{mg}, 78 \%)$; IR [ $\left.\mathrm{cm}^{-1}\right]$ (neat) diastereomer A, 3285, 1640, $1603,1578,1533,1489,1454,1342$; diastereomer B, 3291, 1639, 1603, 1578, 1528, 1489, 1454, 1330; ${ }^{1} \mathrm{H}$ NMR $\left(\mathrm{CDCl}_{3}\right)$ diastereomer $\mathrm{A}, \delta=1.65(\mathrm{~m}, 1 \mathrm{H}), 1.7-1.8(\mathrm{~m}, 2 \mathrm{H})$, $1.98(\mathrm{ddd}, 1 \mathrm{H}, J=14.4,6.4,2.4 \mathrm{~Hz}), 2.21(\mathrm{~s}, 3 \mathrm{H}), 2.6-2.8(\mathrm{~m}, 2 \mathrm{H}), 3.19(\mathrm{mbr}, 1 \mathrm{H}), 5.60$ (ddd, 1H, $J=9.0,6.3,3.6 \mathrm{~Hz}), 7.2-7.4(\mathrm{~m}, 3 \mathrm{H}), 7.27(\mathrm{~m}, 2 \mathrm{H}), 7.42(\mathrm{~m}, 2 \mathrm{H}), 7.49(\mathrm{~m}$, 1H), $7.82(\mathrm{~d}, 2 \mathrm{H}, J=7.6 \mathrm{~Hz}), 8.81(\mathrm{dbr}, 1 \mathrm{H}, J=8.8 \mathrm{~Hz})$; diastereomer $\mathrm{B}, \delta=1.65(\mathrm{~m}$, 1H), 1.8-1.9 (m, 2H), $1.98(\mathrm{~m}, 1 \mathrm{H}), 2,17(\mathrm{~s}, 3 \mathrm{H}), 2.6-2.8(\mathrm{~m}, 2 \mathrm{H}), 2.62(\mathrm{ddd}, 1 \mathrm{H}, J=$ 13.7, 9.8, 6.4 Hz), $2.73(\mathrm{ddd}, 1 \mathrm{H}, J=13.6,10.0,5.6 \mathrm{~Hz}), 2.95(\mathrm{~m}, 1 \mathrm{H}), 5.46(\mathrm{ddbr}, 1 \mathrm{H}$, $J=15.4,7.8 \mathrm{~Hz}), 7.1-7.2(\mathrm{~m}, 3 \mathrm{H}), 7.24(\mathrm{~m}, 2 \mathrm{H}), 7.30(\mathrm{dbr}, 1 \mathrm{H}, J=7.6 \mathrm{~Hz}), 7.43(\mathrm{~m}$, 
$2 \mathrm{H}), 7.51(\mathrm{~m}, 1 \mathrm{H}), 7.82(\mathrm{~d}, 2 \mathrm{H}, J=8.0 \mathrm{~Hz}) ;{ }^{13} \mathrm{C} \mathrm{NMR}\left(\mathrm{CDCl}_{3}\right)$ diastereomer $\mathrm{A}, \delta=$ $14.4,32.3,41.5,41.7,48.8,55.3,125.9,127.0,128.2,128.4,128.4,131.5,133.9,141.5$, 166.3; diastereomer $\mathrm{B}, \delta=13.8,32.2,40.0,42.8,49.1,54.3,63.6,125.9,127.0,127.3$, $128.2,128.4,128.5,131.7,133.8,141.5,166.8 ;$ EI-HRMS $(\mathrm{m} / \mathrm{z})$ calcd. for $\mathrm{C}_{19} \mathrm{H}_{24} \mathrm{~N}_{2} \mathrm{OS}\left(\mathrm{M}^{+}\right)$: 328.1609; diastereomer A, found: 328.1613; diastereomer $\mathrm{B}$, found: 328.1609 .

$N$-Benzyl- $N$-(3-acetamido-5-phenylpentyl)acetamide (7): To a suspension of $\mathrm{LiAlH}_{4}$ (31 mg, $0.817 \mathrm{mmol})$ in THF $(1 \mathrm{~mL})$ was added $6(17.7 \mathrm{mg}, 0.0539 \mathrm{mmol})$ at $\mathrm{rt}$, and the mixture was refluxed for $6 \mathrm{~h}$. After cooled to rt, water $(0.03 \mathrm{~mL}), 15 \%$ aqueous $\mathrm{NaOH}$ solution $(0.03 \mathrm{~mL})$, water $(0.09 \mathrm{~mL})$ were added successively. The mixture was filtrated with Celite ${ }^{\circledR}$, and the collected solid was washed well with $\mathrm{CH}_{2} \mathrm{Cl}_{2}$. The filtrate was concentrated under reduced pressure, and the residue was treated with an excess amount of acetic anhydride in pyridine at $\mathrm{rt}$. The reaction was quenched with a saturated $\mathrm{NaHCO}_{3}$ solution, and $\mathrm{CH}_{2} \mathrm{Cl}_{2}$ was added. The organic phase was separated, and the aqueous phase was extracted with $\mathrm{CH}_{2} \mathrm{Cl}_{2}$. The organic phases were then combined and dried over anhydrou $\mathrm{Na}_{2} \mathrm{SO}_{4}$. The crude product was purified with preparative thin-layer chromatography (silica gel, $\mathrm{CHCl}_{3}: \mathrm{EtOH}=20: 1$ ) to afford the desired product 7 (18.1 mg, 95\%). IR [cm-1] (neat) 3289, 1643, 1552, 1496, 1453, 1374 , $1291 ;{ }^{1} \mathrm{H} \mathrm{NMR}\left(\mathrm{CDCl}_{3}\right.$, two rotamers mixture $) \delta=1.5-1.8(\mathrm{~m}, 4 \mathrm{H}), 1.89(\mathrm{~s}, 0.7 \mathrm{H})$, $1.95(\mathrm{~s}, 2.3 \mathrm{H}), 2.11(\mathrm{~s}, 2.3 \mathrm{H}), 2.14(\mathrm{~s}, 0.7 \mathrm{H}), 2.60(\mathrm{~m}, 2 \mathrm{H}), 3.2(\mathrm{~m}, 1.3 \mathrm{H}), 3.58(\mathrm{~m}, 0.7$ H), $3.90(\mathrm{~m}, 1 \mathrm{H}), 4.48(\mathrm{~d}, 0.7 \mathrm{H}, J=16.8 \mathrm{~Hz}), 4.53(\mathrm{~d}, 0.7 \mathrm{H}, J=16.8 \mathrm{~Hz}), 4.54(\mathrm{~d}$, $0.35 \mathrm{H}, J=14.9 \mathrm{~Hz}), 4.60(\mathrm{~d}, 0.35 \mathrm{H}, J=14.9 \mathrm{~Hz}), 5.18(\mathrm{br}, 0.3 \mathrm{H}), 5.90(\mathrm{dbr}, 0.7 \mathrm{H}, J=$ 8.3 Hz), 7.1-7.4 (m, $10 \mathrm{H}) ;{ }^{13} \mathrm{C} \mathrm{NMR}\left(\mathrm{CDCl}_{3}\right.$, two rotamers mixture $) \delta=21.4,21.8$, $23.3,23.4,32.3,32.4,34.6,36.8,36.9,43.1,45.2,47.4,47.5,48.4,52.5,125.9,126.1$, 
$126.3,127.4,127.7,128.1,128.2,128.3,128.4,128.6,128.6,129.0,136.5,137.6,141.2$, 141.7, 169.8, 170.0, 170.4, 171.2; EI-HRMS (m/z) calcd. for $\mathrm{C}_{22} \mathrm{H}_{28} \mathrm{~N}_{2} \mathrm{O}_{2}\left(\mathrm{M}^{+}\right)$: 352.2151; found: 352.2154 .

1-Acetyl-5-(2-phenylethyl)-2-pyrazoline (8): To a suspension of $\mathrm{LiAlH}_{4}(12.7 \mathrm{mg}$, $0.335 \mathrm{mmol})$ in THF $(0.5 \mathrm{~mL})$ was added $5 \mathbf{5 a c}(105 \mathrm{mg}, 0.274 \mathrm{mmol})$ at $-78{ }^{\circ} \mathrm{C}$, and the mixture was stirred for $9 \mathrm{~h}$ at the same temperature. Water $(0.015 \mathrm{~mL}), 15 \%$ aqueous $\mathrm{NaOH}$ solution $(0.03 \mathrm{~mL})$, water $(0.09 \mathrm{~mL})$ were then added successively to stop the reaction. After warmed to $\mathrm{rt}$, anhydrous $\mathrm{Na}_{2} \mathrm{SO}_{4}$ was added to the mixture, and the whole was stirred for 5 min. The mixture was filtrated with Celite ${ }^{\circledR}$, and the collected solid was washed well with $\mathrm{CH}_{2} \mathrm{Cl}_{2}$. The filtrate was concentrated under reduced pressure, and the residue was treated with acetyl chloride (195 $\mu 1,2.74 \mathrm{mmol})$, pyridine (217 mg, $2.74 \mathrm{mmol}$ ), and p-dimethylaminopyridine (DMAP, $6.7 \mathrm{mg}, 0.052 \mathrm{mmol}$ ) in $\mathrm{CH}_{2} \mathrm{Cl}_{2}(2 \mathrm{~mL})$ at $0{ }^{\circ} \mathrm{C}$ to $\mathrm{rt}$. To the mixture was added a saturated $\mathrm{NaHCO}_{3}$ solution, and the organic phase was separated. The aqueous phase was extracted with $\mathrm{CH}_{2} \mathrm{Cl}_{2}$. The organic phases were combined and dried over anhydrous $\mathrm{Na}_{2} \mathrm{SO}_{4}$. The crude product was purified by preparative thin-layer chromatography (silica gel, hexane-ethyl acetate (1:1) and then $\mathrm{CHCl}_{3}$-ethyl acetate (4:1)) to afford the desired product 7 (45.2 $\mathrm{mg}, 76 \%, 95 \%$ ee). $[\alpha]_{\mathrm{D}}^{28}+227\left(\mathrm{c} 0.564, \mathrm{CHCl}_{3}, 95 \%\right.$ ee); IR $\left[\mathrm{cm}^{-1}\right]$ (neat) 1658, 1600, 1496, 1413, 1357, 1282; ${ }^{1} \mathrm{H}$ NMR $\left(\mathrm{CDCl}_{3}\right) \delta=1.84(\mathrm{~m}, 1 \mathrm{H}), 2.27(\mathrm{~s}, 3 \mathrm{H}), 2.30(\mathrm{~m}, 1 \mathrm{H})$, 2.5-2,7 (m, 3H), $3.02(\mathrm{ddd}, 1 \mathrm{H}, J=18.7,11.2,1.7 \mathrm{~Hz}), 4.46(\mathrm{~m}, 1 \mathrm{H}), 6.85(\mathrm{tbr}, 1 \mathrm{H}, J=$ $1.7 \mathrm{~Hz}), 7.1-7.3(\mathrm{~m}, 5 \mathrm{H}) ;{ }^{13} \mathrm{C} \mathrm{NMR}\left(\mathrm{CDCl}_{3}\right) \delta=22.0,31.0,34.4,39.3,54.4,126.0$, 128.3, 128.4, 140.9, 146.3, 169.2; EI-HRMS (m/z) calcd. for $\mathrm{C}_{13} \mathrm{H}_{16} \mathrm{~N}_{2} \mathrm{O}\left(\mathrm{M}^{+}\right)$: 
216.1263; found: 216.1257; HPLC Daicel Chiralpak AD, hexane $/ \mathrm{PrOH}=40 / 1$, flow rate $=1.0 \mathrm{~mL} / \mathrm{min}: t_{\mathrm{R}}=20 \min ($ major $), t_{\mathrm{R}}=26 \mathrm{~min}($ minor $)$.

S-Ethyl (5-(2-phenylethyl)-2-(p-nitrobenzoyl)pyrazolidin-3-yl)ethanethioate (9): To a solution of 5ac $(58.2 \mathrm{mg}, 0.152 \mathrm{mmol})$ and the trimethylsilyl enol ether of $S$-ethyl ethanethioate $(79.3 \mathrm{mg}, 0.450 \mathrm{mmol})$ in $\mathrm{CH}_{3} \mathrm{CN}(0.5 \mathrm{~mL})$ was added $\mathrm{Me}_{3} \mathrm{SiOTf}(100.1$ $\mathrm{mg}, 0.450 \mathrm{mmol})$ in $\mathrm{CH}_{3} \mathrm{CN}(0.2 \mathrm{~mL})$ at $0{ }^{\circ} \mathrm{C}$, and the whole was stirred for $24 \mathrm{~h}$ at the same temperature. Water was added to quench the reaction, and $\mathrm{CH}_{2} \mathrm{Cl}_{2}$ was added. The organic phase was separated, and the aqueous phase was extracted with $\mathrm{CH}_{2} \mathrm{Cl}_{2}$. The organic phases were combined and dried over anhydrous $\mathrm{Na}_{2} \mathrm{SO}_{4}$. After filtration and concentration under reduced pressure, the crude product was purified by preparative thin-layer chromatography (hexane-ethyl acetate $=3: 1$ ) to afford the desired product 9 $(44.2 \mathrm{mg}, 68 \%, \mathrm{dr}=86 / 14) . \mathrm{IR}\left[\mathrm{cm}^{-1}\right]$ (neat) $3248,1684,1627,1599,1523,1497,1454$, 1413, 1350, 1265, 1228; ${ }^{1} \mathrm{H} \mathrm{NMR}\left(\mathrm{CDCl}_{3}\right.$, two diastereomers mixture $) \delta=1.26(\mathrm{t}, 3 \mathrm{H}$, $J=7.6 \mathrm{~Hz}), 1.4-1.5(\mathrm{~m}, 1.6 \mathrm{H}), 1.6-1.7(\mathrm{~m}, 0.1 \mathrm{H}), 1.7-1.9(\mathrm{~m}, 0.2 \mathrm{H}), 2.1-2.2(\mathrm{~m}, 1.6 \mathrm{H})$, 2.3-2.4 (m, 1.6H), $2.52(\mathrm{~m}, 0.1 \mathrm{H}), 2.65(\mathrm{t}, 0.2 \mathrm{H}, J=7.6 \mathrm{~Hz}), .2 .90(\mathrm{q}, 2 \mathrm{H}, J=7.6 \mathrm{~Hz})$, 3.1-3.2 (m, 1.8H), 3.2-3.3 (m, 0.8H), $4.19(\mathrm{dbr}, 0.1 \mathrm{H}, J=13.2 \mathrm{~Hz}), 4.72(\mathrm{~m}, 1.9 \mathrm{H}), 6.94$ $(\mathrm{d}, 1.8 \mathrm{H}, J=7.6 \mathrm{~Hz}), 7.08(\mathrm{~d}, 0.2 \mathrm{H}, J=7.6 \mathrm{~Hz}), 7.1-7.3(\mathrm{~m}, 3 \mathrm{H}), 7.82(\mathrm{~d}, 0.2 \mathrm{H}, J=8.6$ $\mathrm{Hz}), 7.87(\mathrm{~d}, 1.8 \mathrm{H}, J=8.6 \mathrm{~Hz}), 8.2-8.3(\mathrm{~m}, 2 \mathrm{H}) ;{ }^{13} \mathrm{C} \mathrm{NMR}\left(\mathrm{CDCl}_{3}\right.$, two diastereomers mixture) $\delta=14.6,14.6,23.5,32.7,32.9,33.3,34.0,37.7,39.0,45.9,46.0,53.8,55.5$, $58.7,60.2,122.7,122.7,125.9,126.1,128.2,128.3,128.4,129.7,129.8,140.7,141.0$, 141.4, 141.6, 148.4, 167.5, 168.7, 197.8, 198.0; EI-HRMS $(\mathrm{m} / \mathrm{z})$ calcd. for $\mathrm{C}_{22} \mathrm{H}_{25} \mathrm{~N}_{3} \mathrm{O}_{4} \mathrm{~S}\left(\mathrm{M}^{+}\right)$: 427.1566; found: 427.1548 .

1-Nicotinoyl-5-phenylthiomethyl-2-pyrazoline (10): To a suspension of $\mathrm{LiAlH}_{4}(118$ $\mathrm{mg}, 3.11 \mathrm{mmol})$ in THF (2 mL) was added $5 \mathrm{nc}(617 \mathrm{mg}, 1.54 \mathrm{mmol})$ in THF (2 ml) at 
$-78{ }^{\circ} \mathrm{C}$, and the whole was stirred for $16 \mathrm{~h}$ at the same temperature. Water $(0.12 \mathrm{~mL})$, $15 \%$ aqueous $\mathrm{NaOH}$ solution $(0.12 \mathrm{~mL})$, water $(0.36 \mathrm{~mL})$ were then added successively to stop the reaction. After warmed to rt, anhydrous $\mathrm{Na}_{2} \mathrm{SO}_{4}$ was added to the mixture, and the whole was stirred for 5 min. The mixture was filtrated with Celite ${ }^{\circledR}$, and the collected solid was washed well with $\mathrm{CH}_{2} \mathrm{Cl}_{2}$. The filtrate was concentrated under reduced pressure, and the residue was treated with nicotinoyl chloride hydrochloride (1.10 g, $6.15 \mathrm{mmol}),{ }^{i} \operatorname{Pr}_{2} \mathrm{NEt}(1.59 \mathrm{~g}, 12.3 \mathrm{mmol})$, and $p$-dimethylaminopyridine (DMAP, $187 \mathrm{mg}, 1.53 \mathrm{mmol})$ in $\mathrm{CH}_{2} \mathrm{Cl}_{2}(2 \mathrm{~mL})$ at $0{ }^{\circ} \mathrm{C}$ to rt. To the mixture was added a saturated $\mathrm{NaHCO}_{3}$ solution, and the organic phase was separated. The aqueous phase was extracted with $\mathrm{CH}_{2} \mathrm{Cl}_{2}$. The organic phases were combined and dried over anhydrous $\mathrm{Na}_{2} \mathrm{SO}_{4}$. The crude product was purified with column chromatography (silica gel, hexane-ethyl acetate (1:2)) to afford the desired product $\mathbf{1 0}(312 \mathrm{mg}, 68 \%)$. IR $\left[\mathrm{cm}^{-1}\right]$ (neat) $1635,1604,1587,1481,1435,1338,1284,1257 ;{ }^{1} \mathrm{H} \mathrm{NMR}\left(\mathrm{CDCl}_{3}\right) \delta$ $=2.99(\mathrm{ddd}, 1 \mathrm{H}, J=18.8,5.1,1.7 \mathrm{~Hz}), 3.11(\mathrm{ddd}, 1 \mathrm{H}, J=18.8,10.8,1.4 \mathrm{~Hz}), 3.19(\mathrm{dd}$, $1 \mathrm{H}, J=14.1,8.3 \mathrm{~Hz}), 3.62(\mathrm{dd}, 1 \mathrm{H}, J=13.9,2.7 \mathrm{~Hz}), 4.86(\mathrm{~m}, 1 \mathrm{H}), 6,97$ (sbr, 1H), 7.16 $(\mathrm{t}, 1 \mathrm{H}, J=7.3 \mathrm{~Hz}), 7.2-7.3(\mathrm{~m}, 3 \mathrm{H}), 7.47(\mathrm{~d}, 2 \mathrm{H}, J=7.2 \mathrm{~Hz}), 7.99$ (ddd, $1 \mathrm{H}, J=8.0,1.7$, $1.7 \mathrm{~Hz}), 8.64(\mathrm{dd}, 1 \mathrm{H}, J=4.9,1.5 \mathrm{~Hz}), 8.92(\mathrm{~d}, 1 \mathrm{H}, J=1,4 \mathrm{~Hz}) ;{ }^{13} \mathrm{C} \mathrm{NMR}\left(\mathrm{CDCl}_{3}\right) \delta=$ $34.8,38.6,54.9,122.4,126.3,129.0,129.9,134.5,137.0,148.0,150.5,151.3,165.0$; EI-HRMS (m/z) calcd. for $\mathrm{C}_{16} \mathrm{H}_{15} \mathrm{~N}_{3} \mathrm{OS}\left(\mathrm{M}^{+}\right)$: 297.0936; found: 297.0945 .

(S)-5-Methyl-1-nicotinoyl-2-pyrazoline (ent MS-153, 11): To a suspension of Raney $\mathrm{Ni}(\mathrm{W}-2$, ca $0.5 \mathrm{~g})$ in a mixed solvent $(\mathrm{EtOH}$ and acetate buffer $(\mathrm{pH}=5.2)(2: 1))$ was dissolved compound $10(47.4 \mathrm{mg}, 0.189 \mathrm{mmol})$, and the whole was stirred for $64 \mathrm{~h}$ under hydrogen atmosphere $(1 \mathrm{~atm})$. The mixture was filtered with Celite ${ }^{\circledR}$, and the collected solid was washed well with EtOH. The filtrate was concentrated under 
reduced pressure, and the residue was dissolved in $\mathrm{CH}_{2} \mathrm{Cl}_{2}$. The organic phase was washed with a saturated $\mathrm{NaHCO}_{3}$ solution, and then dried over anhydrous $\mathrm{Na}_{2} \mathrm{SO}_{4}$. After filtration and concentration, the crude mixture was purified by preparative thinlayer chromatography (silica gel, ethyl acetate) to afford the desired product $\mathbf{1 1}(8.6 \mathrm{mg}$, $29 \%, 88 \%$ ee). $[\alpha]_{\mathrm{D}}^{19}+301$ (c $1.16, \mathrm{EtOH}, 91 \%$ ee), lit. $)^{3}[\alpha]_{\mathrm{D}}^{25}-345$ (c $0.5, \mathrm{EtOH}, R-$ form); IR $\left[\mathrm{cm}^{-1}\right]$ (neat) $1639,1593,1570,1479,1435,1336,1284,1255 ;{ }^{1} \mathrm{H}$ NMR $\left(\mathrm{CDCl}_{3}\right) \delta=1.42(\mathrm{~d}, 3 \mathrm{H}, J=6.6 \mathrm{~Hz}), 2.54(\mathrm{ddd}, 1 \mathrm{H}, J=18.6,4.9,2.0 \mathrm{~Hz}), 3.14(\mathrm{ddd}$, $1 \mathrm{H}, J=18.6,10.7,1.7 \mathrm{~Hz}), 4.69(\mathrm{~m}, 1 \mathrm{H}), 6.93(\mathrm{dd}, 1 \mathrm{H}, J=2.0,1.7 \mathrm{~Hz}), 7.31(\mathrm{ddd}, 1 \mathrm{H}$, $J=8.0,4.9,0.8 \mathrm{~Hz}), 8.10(\mathrm{ddd}, J=8.0,2.0,2.0 \mathrm{~Hz}), 8.63(\mathrm{dd}, 1 \mathrm{H}, J=4.9,1.5 \mathrm{~Hz}), 9,02$ $(\mathrm{d}, 1 \mathrm{H}, J=1.7 \mathrm{~Hz}) ;{ }^{13} \mathrm{C} \mathrm{NMR}\left(\mathrm{CDCl}_{3}\right) \delta=19.8,41.3,51.5,122.5,130.6,137.0,147.8$ 150.6, 151.2, 164.8; EI-HRMS (m/z) calcd. for $\mathrm{C}_{10} \mathrm{H}_{11} \mathrm{~N}_{3} \mathrm{O}\left(\mathrm{M}^{+}\right)$: 189.0902; found: 189.0909; HPLC Daicel Chiralcel OD, hexane $/{ }^{\mathrm{PrOH}}=9 / 1$, flow rate $=1.0 \mathrm{~mL} / \mathrm{min}: t_{\mathrm{R}}$ $=27 \min (R), t_{\mathrm{R}}=31 \min (S)$.

\section{References}

1) (a) Cox, P. J.; Wang, W.; Snieckus, V. Tetrahedron Lett. 1992, 33, 2253.

(b) Yamashita, Y.; Ishitani, H.; Shimizu, H.; Kobayashi, S. J. Am. Chem. Soc. 2002, $124,3292$.

2) Kaya, R.; Beller, N. R. J. Org. Chem. 1981, 46, 196.

3) Kajiya, S.; Iizuka, H.; Okumura, K.; Fujiwara, J.; Ohto, N.; Kawazura, H.; Takahashi, Y.; Shiga, Y. Eur. Pat. Appl. 1990, EP373512 A1 19900620. 